\title{
Histological differentiation of metastatic renal carcinoma in the cerebellum from cerebellar haemangioblastoma in von Hippel-Lindau's disease
}

\author{
C A CLELLAND, C S TREIP \\ From the Department of Histopathology, John Bonnet Laboratories, Addenbrookes Hospital, Cambridge, UK
}

SUMMARY Two cases of von Hippel-Lindau's disease with special reference to the occurrence of renal carcinoma are presented. The first case demonstrates the difficulty of differentiating cerebellar haemangioblastoma from metastatic renal carcinoma affecting the cerebellum. The valuable differentiating histological features were positive staining of metastatic renal carcinoma by antiepithelial membrane antigen (anti-EMA) and the demonstration of a distinct pattern of packeting of cells by staining reticulin fibres. Staining with periodic acid Schiff and cytokeratin antibody (anti-CK) were not found to be useful. The second case exhibits the wide variety of neoplasms which may be present in von Hippel-Lindau's disease. Special stains support the findings of the first case.

Von Hippel-Lindau's disease is an autosomal dominant condition with incomplete penetrance and variable expression. ${ }^{1}$ It includes a number of neoplasms, most commonly retinal angioma (von Hippel's tumour), cerebellar haemangiomablastoma (Lindau's tumour), renal cysts, renal carcinoma and phaeochromocytoma. Renal cysts occur in $60 \%$ and renal carcinoma in $45 \%$ of cases. ${ }^{1}$ Other less common components of von Hippel-Lindau's disease include capillary naevi, pancreatic cysts and epididymal cysts. In addition, there are recognised associated conditions such as syringomyelia and erythrocythaemia. ${ }^{2}$

Cerebellar haemangioblastoma accounts for $1 \cdot 1$ to $2.4 \%$ of all intracranial tumours. ${ }^{3}$ Only a minority $(10-20 \%)$ are associated with the syndrome of von Hippel-Lindau's disease and in about $20 \%$ of cases there is a demonstrable familial pattern. ${ }^{2}$ It is important to recognise this uncommon condition because of its inheritance and lethal complications. The expression of the disease is usually delayed until the 3rd or 4th decade; the mean age of presentaton is 35 years. ${ }^{1}$ The initial symptoms are usually either visual disturbances related to retinal angiomas or headaches, nausea, vomiting and vertigo related to cerebellar haemangioblastoma. After the initial presentation other components of the disease may become evident

Address for reprint requests: Dr C A Clelland, Department of Histopathology, John Bonnett Laboratories, Addenbrookes Hospital, Cambridge, England.

Received 8 April 1988 and in revised form 28 July 1988. Accepted 3 September 1988 over the next $10-15$ years. Death is most commonly due to the complications of cerebellar haemangioblas-o toma or metastatic renal carcinoma; the mean age at death is 44.5 years. ${ }^{\prime}$

\section{Case reports}

Case 1

Clinical history and pathological findings A 42 year old married woman presented in 1973 with the symptoms and signs of obstructive hydrocephalus. Bilateral posterior fossa haemangioblastomas were eventually discovered and both were excised at operation in April 1973. Microscopy was typical of cerebellar haemangioblastoma (fig 1). Two daughters have renal cysts identified by abdominal computed tomography (CT). The patient remained well until mid-1977 when further symptoms led to the demonstration of bilateral cerebellar haemangioblastomas; removed at operation in January 1978. Microscopy again confirmed the clinical diagnosis. She was readmitted in December 1979 with further headaches and angiography at that time showed a small haemangioblastoma to the right of the midline just above the level of the foramen magnum. This was not operated on but was observed by serial CT. She then remained asymptomatic until April 1984 when raised intracranial pressure recurred. Angiography demonstrated an increase in size of the right sided lesion. This was explored and a solid and cystic mass was removed. The histology was reported as consistent with cerebellar haemangioblastoma.

She remained well during the summer months but then developed numbness and paraesthesiae of the right side of the face and became increasingly unsteady. CT showed recurrence of the right tonsillar lesion which had become much larger and multilobular. Histological examination of 


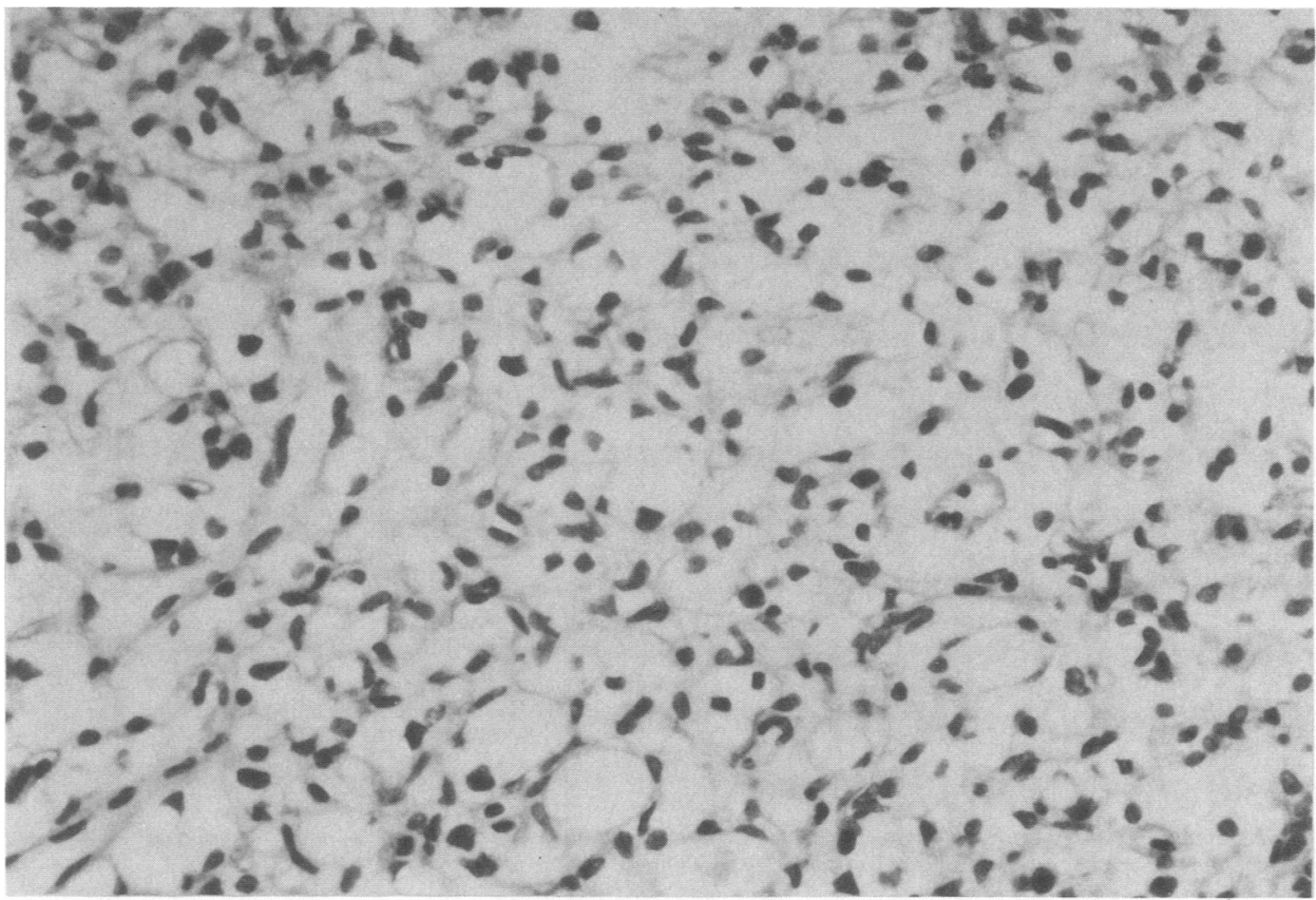

Fig 1 Photomicrograph of $H$ and $E$ stained section of cerebellar tumour (Case 1) consisting of numerous capillaries embedded in a stroma composed of polygonal and spindle cells with abundant foamy cytoplasm typical of a haemangioblastoma. $(\times 360)$.

the mass, which was excised in November 1984 showed a tumour composed of clear cells arranged in an alveolar pattern. The cells contained abundant triglyceride and had nuclei that were not large but showed slight pleomorphism and occasional mitoses (fig 2). The pattern of capillaries seen in the specimens resected in 1973 and 1978 was not conspicuous. The specimen resected in April 1984 showed similarities with the latest histology. The appearance was highly suggestive of metastatic clear cell carcinoma, such as may arise in the kidney.

Investigation of the renal tract by ultrasound revealed a large solid mass with a necrotic centre in the lower pole of the left kidney. CT suggested this lesion was a carcinoma and also demonstrated cysts in the same kidney. The right kidney appeared to be normal. A left nephrectomy was performed in January 1985. At operation, in addition to the tumour of the left kidney, two small cysts were found in the right kidney and a nodule $1.0 \mathrm{~cm}$ across was found in the tail of the pancreas. Histological examination of the tumour in the left kidney demonstrated a clear cell carcinoma. The pancreatic nodule was identified as an islet cell tumour, probably non-secreting. Some tumour cells stained immunocytochemically for insulin and some stained for glucagon but the majority were unstained. There were small deposits of metastatic renal carcinoma in the pancreas. Later in 1985 a retinal angioma was seen. In October 1986 she was admitted to hospital with deterioration in vision and progression of tremor and ataxia with a tendency to fall to the right. CT of the head showed a large lobulated area of enhancement in the right cerebellar hemisphere crossing the mid-line and extending superiorly towards the apex and tentorium. There was dilatation of the 3rd and lateral ventricles and compression of the 4th ventricle. She was treated by radiotherapy with considerable improvement in ataxia.

\section{Histological differentiation of cerebellar haemangioblastoma} from metastatic renal carcinoma

Although in this case the histological diagnosis of metastatic renal carcinoma was not made at the first opportunity (April 1984) the appearances on haematoxylin and eosin stained sections in November 1984 were diagnostic. Histochemical and immunocytochemical staining were carried out on the cerebellar haemangioblastoma, the metastatic renal carcinoma and the primary renal carcinoma. The results are shown in the table.

The reticulin and anti-EMA stains were the most useful of the methods employed. There was clear packeting of small clumps of cells by reticulin fibres of primary and metastatic renal carcinoma. In contrast, the pattern of reticulin fibres in cerebellar haemangioblastoma was diffuse with no packeting. There was strong staining by anti-EMA in the primary and metastatic renal carcinoma (fig 3 ) but no such staining of cerebellar haemangioblastoma. 


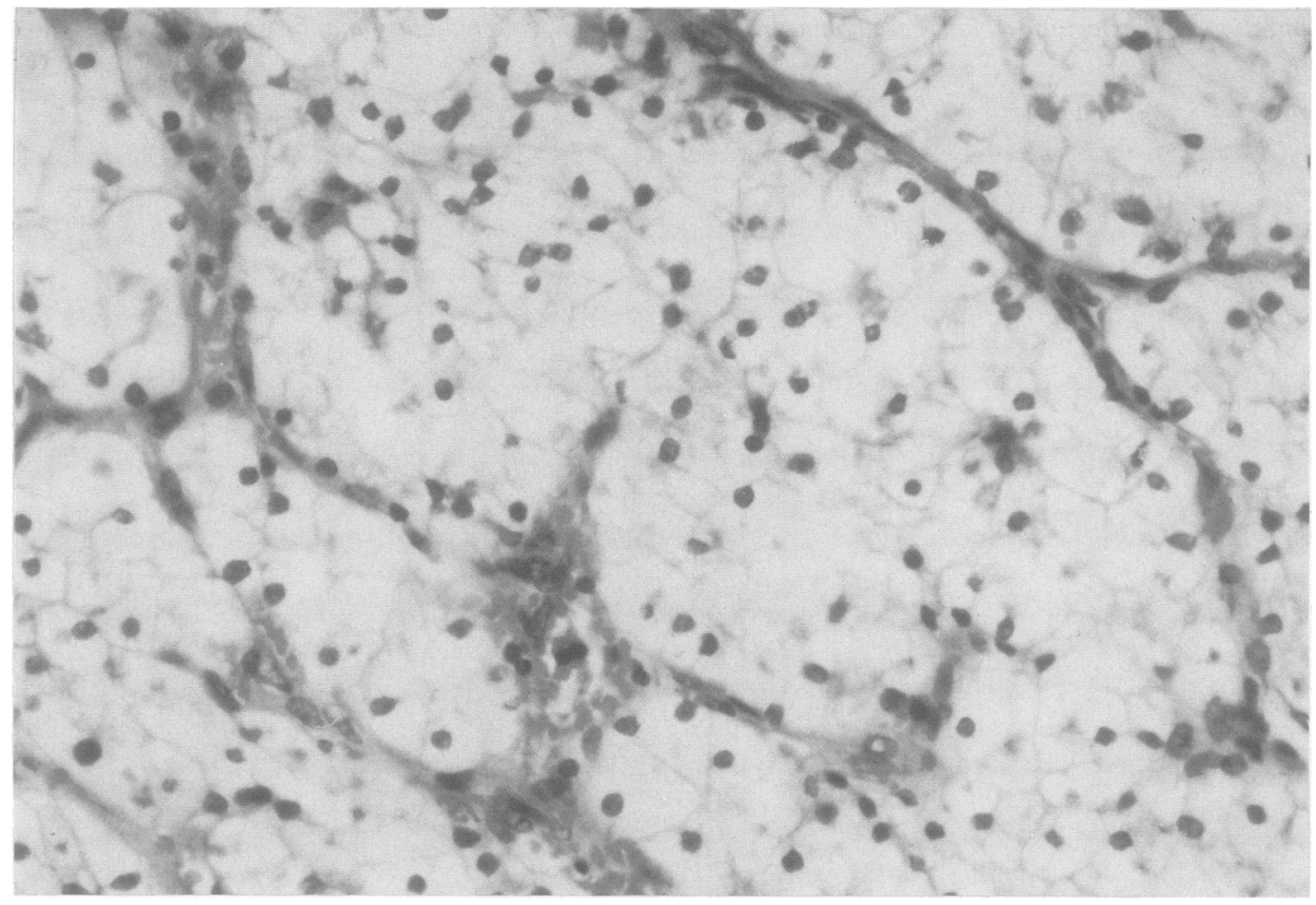

Fig 2 Photomicrograph of $H$ and $E$ stained section of cerebellar tumour (Case 1) composed of nests of polygonal cells with mildly pleomorphic nuclei and clear cytoplasm consistent with metastatic renal carcinoma. $(\times 360)$.

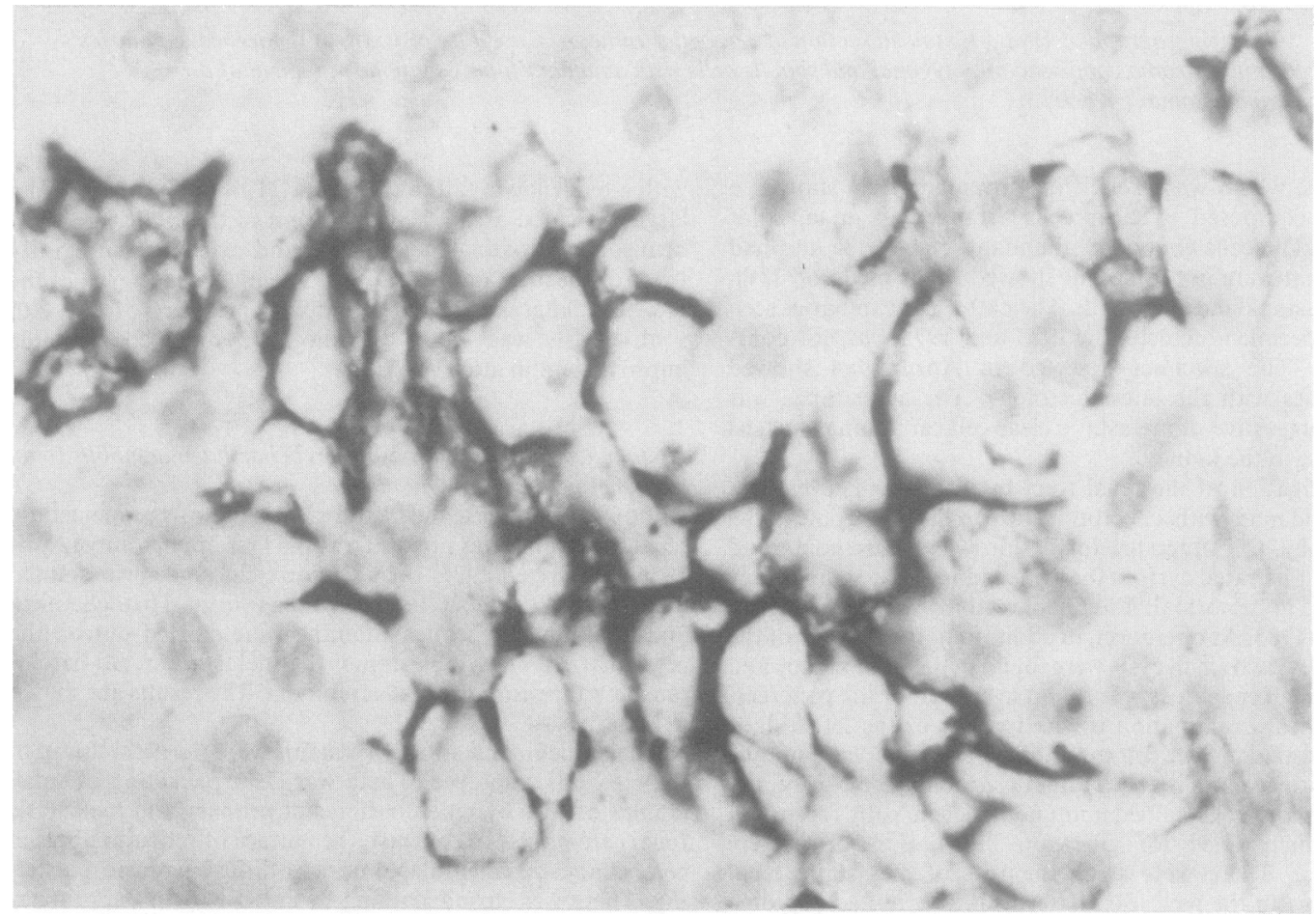

Fig 3 Photomicrograph of EMA stained section of metastatic renal carcinoma from Case 1 showing strong positive staining of cell membranes $(\times 800)$. 

disease

Table Results of special stains in cases 1 and 2

\begin{tabular}{llllll}
\hline & $C K$ & $E M A$ & $P A S$ & Reticulin \\
\hline $\begin{array}{lllll}\text { Case l } \\
\text { Cerebellar haemangioblastoma }\end{array}$ & - & - & + & Diffuse \\
$\begin{array}{l}\text { Metastatic renal carcinoma } \\
\text { Primary renal carcinoma }\end{array}$ & - & + & + & Packeted \\
Case 2 & & & & Packeted \\
$\begin{array}{l}\text { Cerebellar haemangioblastoma } \\
\text { Primary renal carcinoma }\end{array}$ & - & - & - & Diffuse \\
\hline
\end{tabular}

CK-Cytokeratin, EMA-Epithelial membrane antigen, PASPeriodic acid Schin.

\section{Case 2}

Clinical history and pathological findings The patient, a single woman 28 years old in 1968 presented with headache, nausea and vomiting. Vertebral angiography revealed three posterior fossa lesions. Exploration and resection of two of the lesions were performed. Histological examination confirmed the diagnosis of cerebellar haemangioblastoma. She subsequently developed hydrocephalus which required a shunting procedure, followed by revision of the shunt on two occasions. Recurrence of symptoms in 1973 and 1975 necessitated re-exploration and removal of solid and cystic cerebellar tumours. In 1987 she presented with general deterioration due to a large abdominal swelling. Abdominal CT revealed an enormous left ovarian cyst, a smaller right ovarian cyst, a tumour of the left kidney and cysts of the right kidney and pancreas. The ovarian masses and the left renal tumour were removed. The ovaries contained multilocular cysts (the left weighing $2790 \mathrm{~g}$, the right $150 \mathrm{~g}$ ) which histologically proved to be serous adenofibromas. The left kidney contained a well-circumscribed yellow tumour $4.5 \mathrm{~cm}$ in diameter which on histological examination proved to be a typical renal carcinoma. Post-operatively she developed bronchopneumonia and subsequently died.

Post-mortem findings. Death was due to bronchopneumonia and bilateral adrenal haemorrhage with thrombosis of the adrenal veins. In addition to the ovarian and left renal tumours already described, small clear cell carcinomas of the right kidney and a benign papillary cystadenoma of the pancreas were found.

Examination of the fixed brain. There was marked swelling of both cerebral hemispheres with bilateral uncinate grooving. of cystic grey tissue $3.0 \times 3.0 \times 2.0 \mathrm{~cm}$ was present around the medulla and a firm brown mass $2.0 \times 1.0 \times 1.0 \mathrm{~cm}$ was present to the left of the medulla (fig 4). Coronal sections through the cerebral hemispheres showed symmetrical dilatation of the ventricular system with normal cerebral cortex and white matter. Sections of the brain stem showed gross distortion of the pons and medulla. In addition to the tumours already described there were three smaller lesions, one on the wall of the cyst, the other two in the cerebellar cortex. Histological examination showed the grey cystic and brown tumour both to be benign cerebellar haemangioblastomas. Of the smaller lesions the first was a stitch abscess and the other 2 were cerebellar haemangioblastomas. Special stains were performed as for Case 1 on the cerebellar haemangioblastoma and the primary renal carcinoma. The results are shown in the table. As in Case 1 the stains which aided in distinguishing the two tumours were the anti-EMA and the reticulin.

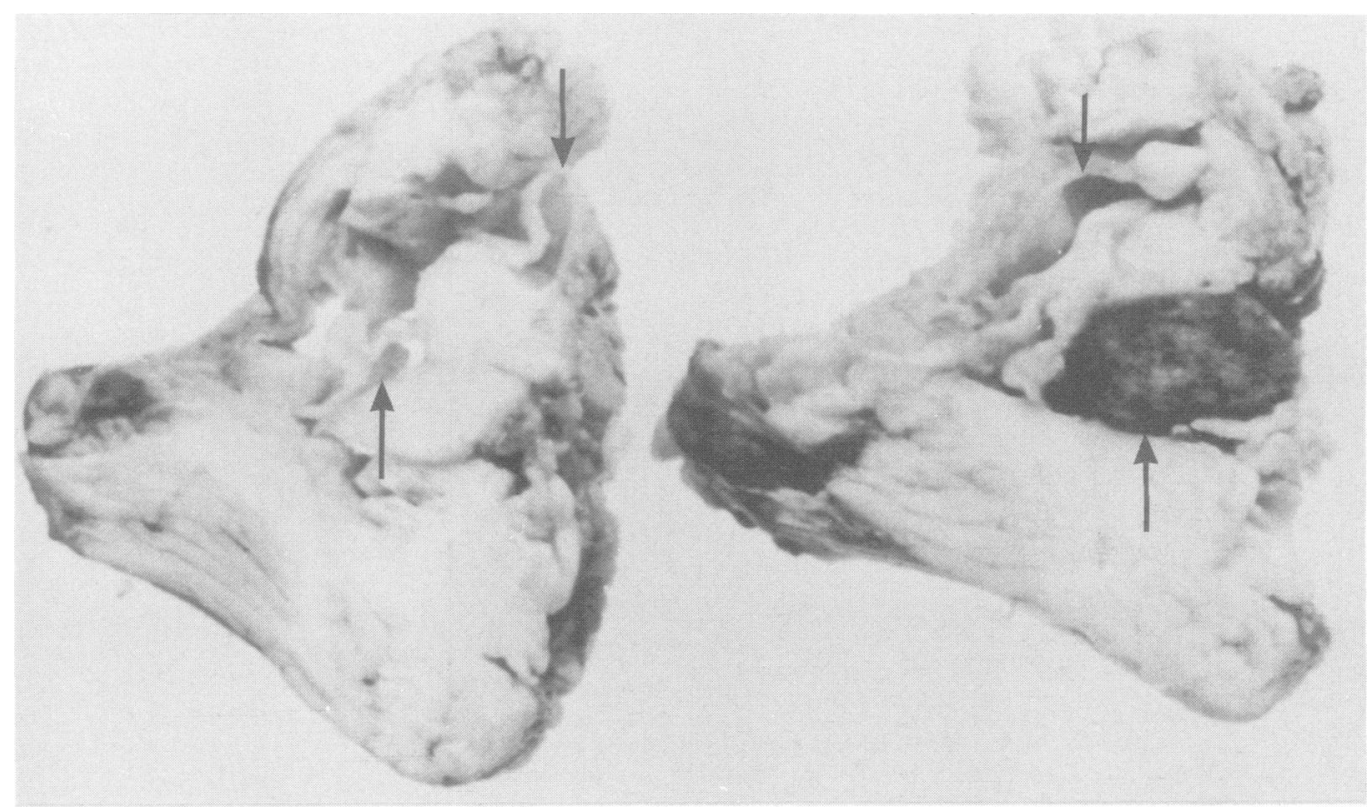

Fig 4 Photograph of $2 \times 1 \mathrm{~cm}$ slices of the brainstem with arrows indicating the solid and cystic haemangioblastomas $(\times 1 \cdot 2)$. 


\section{Discussion}

Renal carcinoma is a common component of von Hippel-Lindau's disease occurring in $45 \%$ of cases ${ }^{1}$ and metastatic disease accounts for a high proportion of deaths. Renal carcinoma peaks between 50 and 70 years in the general population whereas in von HippelLindau's disease the average age at presentation is 41 years.'

The differentiation between cerebellar haemangioblastoma and metastasis from renal carcinoma can be made on routine $\mathrm{H}$ and $\mathrm{E}$ stains in most cases. Where $\mathrm{H}$ and $\mathrm{E}$ and reticulin stain appearances are not discriminatory immunocytochemistry can be of value. Positive staining by anti-EMA will identify metastatic renal carcinoma. ${ }^{4}$ Uniformly negative staining by antiEMA is substantial evidence in favour of a diagnosis of cerebellar haemangioblastoma. Electron microscopy may be helpful in that the finding of microvilli on the tumour cells excludes cerebellar haemangioblastoma and supports the diagnosis of metastatic renal carcinoma. $^{5}$
We thank $\mathrm{Mr} \mathrm{C}$ Burton for producing the photographs and to Mrs B Wilson for the preparation of the histological slides.

\section{References}

1 Christenson PJ, Craig JP, Bibro MC, O'Connell KJ. Cysts containing renal cell carcinoma in von HippelLindau disease. J Urol 1982;128:798-800.

2 Rubenstein LJ. Tumours of the Central Nervous System. Washington: Armed Forces Institute of Pathology, 1972.

3 Zülch KJ. "Biologie und Pathololgie der Hirngeschwulste". In: Olivecrona H, Tonnis W, eds. Handbuch der Neurochirurgie. Berlin: Springer, 1956 vol 3.

4 Andrew SM, Gradwell E. Immunoperoxidase labelled antibody staining in differential diagnosis of central nervous system haemangioblastomas and central nervous system metastases of renal carcinomas. J Clin Pathol 1986;39:917-9.

5 Taxy JB. Renal adenocarcinoma presenting as a solitary metastasis: contribution of electron microscopy to diagnosis. Cancer 1981;48:2056-62. 